\title{
Researches on convex extension problems of fuzzy valued functions
}

\author{
Yu-e Bao*, Bing Dai \\ College of Mathematics, Inner Mongolia University for Nationalities, Inner Mongolia Tongliao 028043, China.
}

\begin{abstract}
In this paper, we extend the concept of fuzzy valued convex functions, subdifferential, and introduce a kind of subdifferential of general fuzzy valued functions. By means of the convexification method, the paper studies the relationships between the subdifferential of general fuzzy valued functions and the subdifferential of convexification fuzzy valued functions, so that we get the conditions of how lower semi continuous fuzzy valued functions can be extended to fuzzy valued convex functions. (C)2016 All rights reserved.
\end{abstract}

Keywords: Fuzzy valued functions, subdifferential, convexification fuzzy valued functions, convex extension.

$2010 M S C$ :

\section{Introduction}

Since the establishment of fuzzy set theory, it has made up for the shortage of classical and statistical mathematics in a certain degree because of its success in dealing with a kind of fuzziness problem which exists widely [1, 7, 11, 14. More and more scholars use the knowledge of fuzzy mathematics to solve practical problems in mathematical programming. And the fuzzy planning developed rapidly because of the joint efforts of the scholars. As it is known to us, the convexity theory in the classical mathematics plays a very important role in mathematical programming [4, 8, 10]. With the development of the research in fuzzy programming, some of the classical mathematical programming methods are naturally considered to be generalized and applied to fuzzy programming. Thus we discuss the convexity of fuzzy sets and the convexity of fuzzy function on convex sets and its application in fuzzy programming [2, 3, 5, 9, 12, which has greatly enriched the contents of

${ }^{*}$ Corresponding author

Email address: byebed@163.com (Yu-e Bao) 
mathematical programming study.

Convex fuzzy programming is a kind of programming problem whose objective function is fuzzy valued convex function (convex fuzzy mapping) and constraint set is convex. In order to use the methods of analytics better to discuss convex fuzzy programming, the concepts of sub gradient the subdifferential are proposed and the extremum problems of fuzzy valued convex functions are discussed, the necessary and sufficient conditions of extremum values for fuzzy valued convex functions are obtained [13, 15]. At the same time, the saddle point and min-max theorem is discussed in the sense of fuzzy set theory in [15], the Lagrange duality and KKT conditions of convex fuzzy programming etc. are also established. The promising applications of fuzzy valued convex function is wide in fuzzy programming, but the fuzzy valued function with convexity is relatively few. Thus, the convex extending of general fuzzy valued functions and the combining of the optimization problem of fuzzy valued function with subdifferential problems of fuzzy valued convex function are meaningful works for us to use the principal of fuzzy valued convex analysis in study of fuzzy optimization problems.

On the convexification problems of general fuzzy valued functions, the method for transforming the general fuzzy valued functions into fuzzy valued convex functions was proposed in [16, 17] via the epigraph of fuzzy valued functions together with the convexification fuzzy valued function characterization theorem. With the help of fuzzy valued functions convexification method, this paper study the convex extension problems of general fuzzy valued function.

\section{Preliminaries}

First, we recall some definitions and results about fuzzy numbers (see [7, 10, 17]). Let $u$ be a fuzzy set that on the real numbers field $\mathrm{R}$, if it has the following properties:

(i) $u$ is normal, i.e., there exists an $x_{0} \in R$ with $u\left(x_{0}\right)=1$;

(ii) $u$ is a fuzzy convex set, i.e. $u(\alpha x+(1-\alpha) y) \geq \min (u(x), u(y))$ whenever $x, y \in R$ and $\alpha \in[0,1]$;

(iii) $u$ is upper semi-continuous;

(iv) $[u]_{0}=\operatorname{cl}\{x \in R \mid u(x)>0\}$ is a compact set.

Let $\mathcal{F}_{0}$ denote the family of fuzzy numbers and $\mathcal{F}_{0}$ is called fuzzy number space. For any $r \in R$, define a fuzzy number $\tilde{r}$ :

$$
\tilde{r}(t)= \begin{cases}1, & t=r \\ 0, & t \neq r\end{cases}
$$

Obviously, $[u]_{\alpha}$ are nonempty bounded closed intervals for every $u \in \mathcal{F}_{0}$ and $\alpha \in[0,1]$, where $[u]_{\alpha}=\left[u_{*}(\alpha), u^{*}(\alpha)\right]$ when $\alpha \in[0,1]$.

We call

$$
u=\left\{\left(u_{*}(\alpha), u^{*}(\alpha), \alpha\right) \mid 0 \leq \alpha \leq 1\right\}
$$

the parametric expression of $u$. A partial order relationship on $\mathcal{F}_{0}$ is defined by

$$
u \leq v \Leftrightarrow u^{*}(\alpha) \leq v^{*}(\alpha), u_{*}(\alpha) \leq v_{*}(\alpha) \text {, for any } \alpha \in[0,1] .
$$

For any $u, v \in \mathcal{F}_{0}$ and $r \in R$, the addition and scalar multiplication on $\mathcal{F}_{0}$ can be represented as:

$$
\begin{aligned}
u+v & =\left\{\left(u_{*}(\alpha), u^{*}(\alpha), \alpha\right) \mid 0 \leq \alpha \leq 1\right\}+\left\{\left(v_{*}(\alpha), v^{*}(\alpha), \alpha\right) \mid 0 \leq \alpha \leq 1\right\} \\
& =\left\{\left(u_{*}(\alpha)+v_{*}(\alpha), u^{*}(\alpha)+v^{*}(\alpha), \alpha\right) \mid 0 \leq \alpha \leq 1\right\},
\end{aligned}
$$




$$
r u=\left\{\left((r u)_{*}(\alpha),(r u)^{*}(\alpha), \alpha\right) \mid 0 \leq \alpha \leq 1\right\} .
$$

Where

$$
(u+v)_{*}(\alpha)=u_{*}(\alpha)+v_{*}(\alpha),(u+v)^{*}(\alpha)=u^{*}(\alpha)+v^{*}(\alpha), 0 \leq \alpha \leq 1 .
$$

If $r \geq 0$,

$$
(r u)_{*}(\alpha)=r u_{*}(\alpha),(r u)^{*}(\alpha)=r u^{*}(\alpha), 0 \leq \alpha \leq 1
$$

and if $r<0$

$$
(r u)_{*}(\alpha)=r u^{*}(\alpha),(r u)^{*}(\alpha)=r u_{*}(\alpha), 0 \leq \alpha \leq 1 .
$$

For $\xi_{i}(i=1,2, \cdots, n) \in \mathcal{F}_{0}$, we call $\xi=\left(\xi_{1}, \xi_{2}, \cdots, \xi_{n},\right)$ is an $n$-dimensional fuzzy vector. Let $\mathcal{F}_{0}^{n}(R)$ denote the set of all $n$-dimensional fuzzy vectors.

Let $R^{n}$ denote $n$-dimensional Euclidean Space. For

$$
\xi=\left(\xi_{1}, \xi_{2}, \cdots, \xi_{n},\right) \in \mathcal{F}_{0}^{n}(R), x=\left(x_{1}, x_{2}, \cdots, x_{n}\right) \in R^{n}
$$

the inner product of $\xi$ and $x$ is defined as

$$
<\xi, x>=x_{1} \xi_{1}+x_{2} \xi_{2}+\cdots+x_{n} \xi_{n} .
$$

Let $D$ be a nonempty subset of $R^{n}$. A mapping $f: D \rightarrow \mathcal{F}_{0}$ is said to be a fuzzy valued function. By the parameter expression of fuzzy number, the fuzzy valued function can be expressed as

$$
f(x)=\left\{\left(f(x)_{*}(\alpha), f(x)^{*}(\alpha), \alpha\right) \mid 0 \leq \alpha \leq 1\right\},
$$

where $[f(x)]^{\alpha}=\left[f(x)_{*}(\alpha), f(x)^{*}(\alpha)\right]$, for every $\alpha \in[0,1]$.

Definition 2.1 ([16]). Let $f: D \rightarrow \mathcal{F}_{0}$ be a fuzzy valued function, $G=c o n v($ epif $)$, then the fuzzy valued function defined on convD

$$
f_{C}(x)=\inf \left\{u \mid(x, u) \in G, u \in \mathcal{F}_{0}\right\}
$$

is called the convex hull of $f$, denoted by $f_{C}=\operatorname{conv} f$. Where

$$
\text { epif }=\left\{(x, u) \mid x \in D, u \in \mathcal{F}_{0}, f(x) \leq u\right\}
$$

is the epigraph of $f$, convD and conv(epif) are the convex hulls corresponding to $D$ and epif, respectively.

In paper [16, 17], it has been proved that $f_{C}=c o n v f$ is the fuzzy valued convex function on conv $D$, and $f_{C}$ is called the convexification fuzzy valued function of $f$.

Theorem 2.2 ([16]). Let $f_{C}$ be the convexification fuzzy valued function of fuzzy valued function $f: D \rightarrow \mathcal{F}_{0}$, then

$$
\begin{aligned}
& f_{C}(x)=\inf _{m \in N}\left\{\lambda_{1} f\left(x_{1}\right)+\lambda_{2} f\left(x_{2}\right)+\cdots+\lambda_{m} f\left(x_{m}\right) \mid \lambda_{i}>0\right. \\
&\left.x_{i} \in D(i=1,2, \cdots, m), \sum_{i=1}^{m} \lambda_{i}=1, \sum_{i=1}^{m} \lambda_{i} x_{i}=x\right\}
\end{aligned}
$$


Definition $2.3([3])$. Let $f: D \rightarrow \mathcal{F}_{0}$ be a fuzzy valued function.

- Let $x_{0} \in D, f$ is lower semi continuous at $x_{0}$ if for every $\varepsilon>0$, there exists an $\delta>0$ so that

$$
f\left(x_{0}\right) \leq f(x)+\tilde{\varepsilon}
$$

for all $x \in D$ and $\left\|x-x_{0}\right\|<\delta$.

- $f$ is said to be lower semi continuous if it is lower semi continuous at every point of $D$.

Property 2.4. If $f$ be the lower semi continuous fuzzy valued function on $D$. Then for any $r \in[0,1]$, $f(x)_{*}(r)$ and $f(x)^{*}(r)$, are lower semi continuous real valued functions on $D$.

Proof. Let $f$ be the lower semi continuous fuzzy valued function on $D, x_{0} \in D$. Then for any $\varepsilon>0$, there exists an $\delta>0$ satisfied $f\left(x_{0}\right) \leq f(x)+\tilde{\varepsilon}$ when

$$
\left\|x-x_{0}\right\|<\delta .
$$

Therefore, for every $r \in[0,1]$, we have

$$
\begin{aligned}
& f\left(x_{0}\right)_{*}(r) \leq f(x)_{*}(r)+\varepsilon, \\
& f\left(x_{0}\right)^{*}(r) \leq f(x)^{*}(r)+\varepsilon .
\end{aligned}
$$

It follows that $f(x)_{*}(r)$ and $f(x)^{*}(r)$ are lower semi continuous fuzzy valued functions on D.

Lemma $2.5\left([\underline{6})\right.$. Let $f: D \rightarrow(-\infty,+\infty)$ be a real valued function, $x_{0} \in D$. Then the following conditions are equivalent:

(1) $f$ is lower semi continuous at point $x_{0}$;

(2) for any $\varepsilon>0$, there exists $\delta>0$, such that when $\left\|x-x_{0}\right\|<\delta$, there has $f\left(x_{0}\right) \leq f(x)+\epsilon$.

(3) for any $x_{n} \in D(n=1,2, \cdots)$, if $\left\|x_{n}-x_{0}\right\| \rightarrow 0(n \rightarrow \infty)$, then $f\left(x_{0}\right) \leq \underline{\lim }_{n \rightarrow \infty} f\left(x_{n}\right)$.

Definition 2.6 ([15]). Let $\xi$ be an $n$-dimensional fuzzy vector, $f: D \rightarrow \mathcal{F}_{0}$ be a fuzzy valued convex function, $x \in D$. If for any $z \in D$, there has

$$
f(z) \geq f(x)+<\xi, z-x>,
$$

then we call $\xi$ the sub gradient of $f$ at $x$.

Call the

$$
\partial f(x)=\left\{\xi \mid \xi \in \mathcal{F}_{0}^{n}(R), f(z) \geq f(x)+<\xi, z-x>, \forall z \in D\right\}
$$

subdifferential of $f$ at $x$.

If $\partial f(x) \neq \phi$, then $f$ is called subdifferentiable at $x$, or called $f$ exists the subdifferential at $x$.

We generalize the concept of fuzzy valued convex functions subdifferential above, and introduce a kind of subdifferential of general fuzzy valued functions which is defined as:

Definition 2.7. Let $f: D \rightarrow \mathcal{F}_{0}$ be a general fuzzy valued function, $x \in D$. The subdifferential of $f$ at $x$ is defined as

$$
\partial f(x)=\left\{\xi \mid \xi \in \mathcal{F}_{0}^{n}(R), f(z) \geq f(x)+<\xi, z-x>, \forall z \in D\right\} .
$$

If $\partial f(x) \neq \phi$, then $f$ is called sub differentiable at $x$, or it is said that the subdifferential of $f$ exists at $x$. 


\section{Main Results}

By means of the convexification method (Definition 2.1), we study the relationships between the subdifferential of general fuzzy valued functions and the subdifferential of convexification fuzzy valued functions, then we get the existence conditions of convex extension for fuzzy valued functions.

Theorem 3.1. Let $f: D \rightarrow \mathcal{F}_{0}$ be a fuzzy valued function. Then $f$ is sub differentiable at $x$ if and only if $f_{C}(x)=f(x)$ and the convexification fuzzy valued function $f_{C}$ of $f$ is also sub differentiable at point $x$.

Proof. Necessity: Let $f$ be sub differentiable at $x$, then there exists $\xi \in \mathcal{F}_{0}^{n}(R)$, such that

$$
f(y) \geq f(x)+<\xi, y-x>\text {, for every } y \in D .
$$

Thus, for $m \in N, \lambda_{i}>0, x_{i} \in D(i=1,2, \cdots, m)$, when $\sum_{i=1}^{m} \lambda_{i}=1, \sum_{i=1}^{m} \lambda_{i} x_{i}=x$, we have

$$
f\left(x_{i}\right) \geq f(x)+<\xi, x_{i}-x>(i=1,2, \cdots, m) .
$$

Therefore,

$$
\sum_{i=1}^{m} \lambda_{i} f\left(x_{i}\right) \geq f(x)+<\xi, \sum_{i=1}^{m} \lambda_{i} x_{i}-x>=f(x)
$$

Thus, we have

$$
\inf _{m \in N}\left\{\sum_{i=1}^{m} \lambda_{i} f\left(x_{i}\right) \mid \lambda_{i}>0, x_{i} \in D(i=1,2, \cdots, m), \sum_{i=1}^{m} \lambda_{i}=1, \sum_{i=1}^{m} \lambda_{i} x_{i}=x\right\} \geq f(x),
$$

that is

$$
f_{C}(x) \geq f(x)
$$

On the other hand, by Theorem 2.2, we know that $f_{C}(x) \leq f(x)$. Hence $f_{C}(x)=f(x)$.

In the following, we prove that $f_{C}$ is sub differentiable at $x$.

For every $y \in \operatorname{conv} D$, let $\lambda_{i}>0(i=1,2, \cdots, m)$ and $\sum_{i=1}^{m} \lambda_{i}=1$, so that

$$
\sum_{i=1}^{m} \lambda_{i} y_{i}=y
$$

Since $f$ is sub differentiable at point $x$, there exists $\xi \in \partial f(x)$ such that

$$
f\left(y_{i}\right) \geq f(x)+<\xi, y_{i}-x>(i=1,2, \cdots, m),
$$

therefore,

$$
\sum_{i=1}^{m} \lambda_{i} f\left(y_{i}\right) \geq f(x)+<\xi, \sum_{i=1}^{m} \lambda_{i} y_{i}-x>=f(x)+<\xi, y-x>
$$

Thus, we have

$$
\begin{aligned}
& \inf _{m \in N}\left\{\sum_{i=1}^{m} \lambda_{i} f\left(y_{i}\right) \mid \lambda_{i}>0, x_{i} \in D(i=1,2, \cdots, m), \sum_{i=1}^{m} \lambda_{i}=1, \sum_{i=1}^{m} \lambda_{i} y_{i}=y\right\} \\
& \quad \geq f(x)+<\xi, y-x>.
\end{aligned}
$$


It follows from $f_{C}(x)=f(x)$, we have

$$
f_{C}(y) \geq f_{C}(x)+<\xi, y-x>.
$$

So $\xi \in \partial f_{C}(x)$. Thereby, $f_{C}$ is sub differentiable at point $x$.

Sufficiency: Let $f_{C}$ be sub differentiable at point $x$, then there exists $\xi \in \mathcal{F}_{0}\left(R^{n}\right)$, for any $y \in \operatorname{conv} D$, such that

$$
f_{C}(y) \geq f_{C}(x)+<\xi, y-x>.
$$

It follows from $f_{C}(x)=f(x)$ and $D \subset \operatorname{conv} D$, we get

$$
f(y) \geq f_{C}(y) \geq f(x)+<\xi, y-x>, \forall y \in D .
$$

Thus we have $\xi \in \partial f(x) \neq \varnothing$, thereby $f$ is sub differentiable at point $x$.

Theorem 3.2. Let $f: D \rightarrow \mathcal{F}_{0}$ be a lower semi continuous fuzzy valued function. If for any convex extreme subset $E(\operatorname{dim} E>0)$ of convD, the set $B$ where the subdifferential exist of $\left.f\right|_{E \cap D}$ is dense in $E \cap D$, then

(1) The convexification fuzzy valued function $f_{C}$ of $f$ is the extension of $f$.

(2) The subdifferential of $\left.f_{C}\right|_{E}$ exists on $B$.

Proof.

(1) Let $x_{0} \in D$. If $x_{0}$ is an extreme point of $D$, obviously we have $f_{C}\left(x_{0}\right)=f\left(x_{0}\right)$. If $x_{0}$ is not an extreme point of $D$, then let $x_{i} \in D, \lambda_{i}>0(i=1,2, \cdots, m)$, and $\sum_{i=1}^{m} \lambda_{i}=1$, such that

$$
\sum_{i=1}^{m} \lambda_{i} x_{i}=x_{0} .
$$

Let $E$ be the minimum convex extreme subset of conv $D$ containing $x_{0}, x_{1}, \cdots, x_{m}$. Since $x_{0}$ is not an extreme point, then we have $\operatorname{dim} E>0$.

Next, we will prove $\operatorname{conv}(E \bigcap D)=E$.

By $E \bigcap D \subset E$, we obtain that

$$
\operatorname{conv}(E \bigcap D) \subset \operatorname{conv} E=E
$$

Conversely, for $y \in E \subset \operatorname{conv} D$, there exist $y_{i} \in D, \beta_{i}>0(i=1,2, \cdots, j)$, and $\sum_{i=1}^{j} \beta_{i}=1$, such that

$$
\sum_{i=1}^{j} \beta_{i} y_{i}=y
$$

Since $E$ is the extreme subset, thus $y_{i} \in E(i=1,2, \cdots, j)$, hence

$$
y \in E \bigcap D(i=1,2, \cdots, j) .
$$

Therefore

$$
y \in \operatorname{conv}(E \bigcap D)
$$

thus, $\operatorname{conv}(E \bigcap D)=E$. 
Next, we prove that $x_{0} \in i \operatorname{cr} E$ (where $i c r E$ is the relative interior point set of $E$ ).

Assume $x_{0} \notin i c r E$, then $x_{0} \in E / i c r E$, by the separation theorem of convex sets, there exists

$$
x^{*} \in R^{n}
$$

such that

$$
<x^{*}, x_{0}>\geq \sup _{y \in E}<x^{*}, y>
$$

hence

$$
<x^{*}, x_{i}>\leq<x^{*}, x_{0}>(i=1,2, \cdots, m) .
$$

It follows from

$$
<x^{*}, \sum_{i=1}^{m} \lambda_{i} x_{i}>=<x^{*}, x_{0}>
$$

we easily obtain

$$
<x^{*}, x_{i}>=<x^{*}, x_{0}>(i=1,2, \cdots, m) .
$$

Let

$$
A=\left\{y \in E \mid<x^{*}, y>=<x^{*}, x_{0}>\right\},
$$

then it is easy to prove $A$ is the convex extreme subset of $E$ and $A \subset E$.

Namely, $A$ is the convex extreme subset of conv $D$ and containing $x_{0}, x_{1}, \cdots, x_{m}$.

This contradicts $E$ is the minimum convex extreme subset of convD containing $x_{0}, x_{1}, \cdots, x_{n}$. Thus, $x_{0} \in i c r E$. Let

$$
g: E \rightarrow \mathcal{F}_{0}(\operatorname{conv}(E \bigcap D)=E)
$$

be the convexification fuzzy valued function of $\left.f\right|_{E \cap D}$, then for any $r \in[0,1], g(x)_{*}(r)$ and $g(x)^{*}(r)$ are the real valued convex functions on $E$ and continuous at $x_{0} \in i \mathrm{cr} E$.

Since set $B$ on which the subdifferential of $\left.f\right|_{E \cap D}$ exists is dense in $E \bigcap D$. Hence, for $x_{0} \in E \bigcap D$, there exists $\left\{z_{n}\right\} \subset B$ such that $z_{n} \rightarrow x_{0}(n \rightarrow \infty)$. By Theorem 3.1, we have

$$
f\left(z_{n}\right)=g\left(z_{n}\right)=\left.f\right|_{E \cap D}\left(z_{n}\right) .
$$

Since $f$ is lower semi continuous, we get $f(x)_{*}(r)$ and $f(x)^{*}(r)$ are lower semi continuous at $x_{0} \in i \operatorname{cr} E$ by Property 2.4. Hence, from Lemma 2.5, for any $r \in[0, r]$, we have

$$
\begin{aligned}
& f\left(x_{0}\right)_{*}(r) \leq \varliminf_{n \rightarrow \infty} f\left(z_{n}\right)_{*}(r)=\lim _{n \rightarrow \infty} g\left(z_{n}\right)_{*}(r)=g\left(x_{0}\right)_{*}(r), \\
& f\left(x_{0}\right)^{*}(r) \leq \varliminf_{n \rightarrow \infty} f\left(z_{n}\right)^{*}(r)=\lim _{n \rightarrow \infty} g\left(z_{n}\right)^{*}(r)=g\left(x_{0}\right)^{*}(r) .
\end{aligned}
$$

We have

$$
f\left(x_{0}\right) \leq g\left(x_{0}\right)
$$

Consequently,

$$
\begin{aligned}
g\left(x_{0}\right) & =\inf _{m \in N}\left\{\sum_{i=1}^{m} \lambda_{i} f\left(x_{i}\right) \mid \lambda_{i}>0, x_{i} \in D \bigcap E(i=1,2, \cdots, m), \sum_{i=1}^{m} \lambda_{i}=1, \sum_{i=1}^{m} \lambda_{i} x_{i}=x_{0}\right\} \\
& \leq \sum_{i=1}^{m} \lambda_{i} f\left(x_{i}\right) .
\end{aligned}
$$


Thereby, we have $f\left(x_{0}\right) \leq \sum_{i=1}^{m} \lambda_{i} f\left(x_{i}\right)$. Hence,

$$
f\left(x_{0}\right) \leq \inf _{m \in N}\left\{\sum_{i=1}^{m} \lambda_{i} f\left(x_{i}\right) \mid \lambda_{i}>0, x_{i} \in D(i=1,2, \cdots, m), \sum_{i=1}^{m} \lambda_{i}=1, \sum_{i=1}^{m} \lambda_{i} x_{i}=x\right\} .
$$

That is, $f\left(x_{0}\right) \leq f_{C}\left(x_{0}\right)$.

On the other hand, $f\left(x_{0}\right) \geq f_{C}\left(x_{0}\right)$. So $f\left(x_{0}\right)=f_{C}\left(x_{0}\right)$. Thus, we have $f_{C}$ is the extension of $f$ by $D \subset \operatorname{conv} D$.

(2) We now prove that the subdifferential of $\left.f_{C}\right|_{E}$ exists on $B$. For every $x \in B$, by the known conditions, we have $\left.\partial f\right|_{E \cap D}(x) \neq \varnothing$. Next, we prove

$$
\left.\left.\partial f_{C}\right|_{E}(x) \supset \partial f\right|_{E \cap D}(x)
$$

For every $y \in E \subseteq \operatorname{conv} D$, let $y_{i} \in D, v_{i}>0(i=1,2, \cdots, k)$, and $\sum_{i=1}^{k} v_{i}=1$, such that $y=$ $\sum_{i=1}^{k} v_{i} y_{i}$. Since $E$ is the extreme subset, thus according to $y_{i} \in E(i=1,2, \cdots, k)$, we have $y_{i} \in D \bigcap E(i=1,2, \cdots, k)$. Hence, for $\left.\xi_{x} \in \partial f\right|_{E \cap D}(x)$, we get

$$
f\left(y_{i}\right) \geq f(x)+<\xi_{x}, y_{i}-x>(i=1,2, \cdots, k) .
$$

Therefore

$$
\sum_{k=1}^{k} v_{i} f\left(y_{i}\right) \geq f(x)+<\xi_{*}, \sum_{k=1}^{k} v_{i} y_{i}-x>(i=1,2, \cdots, k)
$$

Thus, we have

$$
\inf _{k \in N}\left\{\sum_{i=1}^{k} \lambda_{i} f\left(y_{i}\right) \mid v_{i}>0, y_{i} \in D(i=1,2, \cdots, k), \sum_{i=1}^{k} v_{i}=1, \sum_{i=1}^{k} v_{i} x_{i}=y\right\} \geq f(x)+<\xi_{x}, y-x>.
$$

Since $f_{C}(x)=f(x)$ on $B \subset D$. Hence, for any $y \in E$,

$$
f_{C}(y) \geq f_{C}(x)+<\xi_{x}, y-x>.
$$

Hence, $\left.\left.\partial f\right|_{E}(x) \supset \partial f\right|_{E \cap D}(x)$, that is $\left.f\right|_{E}(x) \neq \emptyset$. By the arbitrary of $x$, the subdifferential of $\left.f_{C}\right|_{E}$ exists on $B$.

By Theorem 3.2 , we easily obtain the following corollary:

Corollary 3.3. Let $f: D \rightarrow \mathcal{F}_{0}$ be a lower semi continuous fuzzy valued function and $D$ be a convex set. If the set on which the subdifferential of $\left.f\right|_{E}$ exists is dense in $E$ for any convex extreme subset $E$ of $D$, then $f$ is fuzzy valued convex function.

\section{Conclusions}

It is well known that fuzzy valued function can not always be extended to fuzzy valued convex functions. Hence the convex extension of general fuzzy valued functions is a natural and important problem, which is helpful to improve the efficiency of global optimization methods. In this paper, we generalize the concept of fuzzy valued convex functions subdifferential, and propose a kind of subdifferential of general fuzzy valued functions. By means of the convexification method, we study the relations between the subdifferential of general fuzzy valued functions and the subdifferential of convexification fuzzy valued functions, then we get the existence conditions of convex extension for fuzzy valued functions. These conditions contribute a new method to the study of the optimization problems on how to transform general fuzzy valued functions into convex fuzzy programming. 


\section{Acknowledgments}

This work is supported by the National Science Fund of China (11461052) and the Inner Mongolia Natural Science Foundation of China(2014MS0107).

\section{References}

[1] E. Ammar, J. Metz, On fuzzy convexity and parametric fuzzy optimization, Fuzzy Sets Syst., 49 (1992), 135-141.1.

[2] Y. E. Bao, Some Properties of Subdifferential Convex Fuzzy Mappings, J. Fuzzy math., 21 (2013), 927-936.1

[3] Y. E. Bao, C. X. Wu, Convexity and Semi continuity of Fuzzy Mappings, Comput. Math. Appl., 51 (2006), 1809-1816.1. 2.3

[4] Y. E. Bao, C. X. Wu, On the convexity and strict convexity of fuzzy mapping, J. Harbin Inst. Tech., 4 (2007), 639-641.1.

[5] Y. E. Bao, C. X. Wu, Semi strictly convex fuzzy mappings, J. Math. Res. Expo., 30 (2010), 571-580.1

[6] H. Brezis, Analyse Fonctionnelle: Theorie Et Applications, Dunod, Paris, (2002).2.5

[7] N. Furukawa, Convexity and local Lipschitz continuity of fuzzy-valued mappings, Fuzzy Sets Syst., 93 (1998), 113-119.1. 2

[8] Z. T. Gong, H. X. Li, Derivatives and gradients of fuzzy mappings and their applications, (Chinese), Appl. Math. J. Chinese Univ. Ser. A, 25 (2010), 229-238. 1

[9] J. Lia, M. A. Noor, On properties of convex fuzzy mappings, Fuzzy Sets Syst., 219 (2013), 113-125.1

[10] S. Nanda, K. Kar, Convex Fuzzy Mappings, Fuzzy Sets Syst., 48 (1992), 129-132.1. 2

[11] M. Panigrahi, G. Panda, Sudarsan Nanda, Convex fuzzy mapping with differentiability and its application in fuzzy optimization, European J. Oper. Res., 185 (2008), 47-62.1

[12] Y. R. Syau, Differentiability and Convexity of Fuzzy Mappings, Comput. Math. Appl., 41 (2001), 73-81. 1

[13] G. X. Wang, C. X. Wu, Directional Derivatives and Sub differential of Convex Fuzzy Mappings and Application in Convex Fuzzy Programming, Fuzzy Sets Syst., 138 (2003), 559-591.1.

[14] L. A. Zadeh, Fuzzy Sets, Inf. Control., 8 (1965), 338-353.1]

[15] C. Zang, X. H. Yuan, E. S. Lee., Duality Theory in Fuzzy Mathematical Programming Problems with Fuzzy Coefficients, Comput. Math. Appl., 49 (2005), 1709-1730.1.2.6

[16] C. Zang, X. H. Yuan, E. S. Lee, Convex Fuzzy Mapping and Operations of Convex Fuzzy Mappings, Comput. Math. Appl., 51 (2006), 143-152.1, 2.1, 2.2

[17] B. Zhao, Y. E. Bao, X. Q. Peng, Some operation properties of a class of fuzzy convex functions, (Chinese) Mohu Xitong yu Shuxue, 26 (2012), 167-171.1. 2, 2.1 